\title{
Experiments with a double solenoid system: Measurements of the ${ }^{6} \mathrm{He}+p$ Resonant Scattering
}

\author{
R. Pampa Condori ${ }^{1, a}$, R. Lichtenthäler ${ }^{1, b}$, A. Lépine-Szily ${ }^{1}$, L. R. Gasques ${ }^{1}$, P. N de Faria ${ }^{2}$, D.R. \\ Mendes Jr. ${ }^{2}$, M.C. Morais ${ }^{1}$, K.C.C. Pires ${ }^{3}$, V.B. Scarduelli ${ }^{1}$, E. Leistenschneider ${ }^{1}$, J.A. Alcántara- \\ Núñez ${ }^{1}$, J.M.B. Shorto ${ }^{4}$, and M. Assunção ${ }^{5}$ \\ ${ }^{1}$ Departamento de Física Nuclear, Instituto de Física da Unidersidade de São Paulo, P.O.Box 66318, SP, \\ Brazil \\ ${ }^{2}$ Universidade Federal Fluminense, Centro de Estudos Gerais, Instituto de Física, Niteroi, RJ, Brazil \\ ${ }^{3}$ Universidade Tecnológica Federal do Paraná, Campus Cornélio Procópio, PR, Brazil \\ ${ }^{4}$ Instituto de Pesquisas Energeticas e Nucleares, IPEN/CNEN, São Paulo, Brazil \\ ${ }^{5}$ Universidade Federal de São Paulo, Campus Diadema, São Paulo, SP, Brazil
}

\begin{abstract}
A recent experiment has been performed in the double solenoid system Radioactive Ion Beams in Brasil (RIBRAS) by impinging a pure ${ }^{6} \mathrm{He}$ secondary beam on a thick $\mathrm{CH}_{2}$ target to measure the ${ }^{6} \mathrm{He}+p$ excitation function. Results of this experiment will be presented.
\end{abstract}

\section{Introduction}

The spectroscopy of light nuclei such as Li, Be and others at high excitation energies is a field still not fully explored[1]. The ability to produce those nuclei using reactions induced by exotic nuclei is recent and motivating. In particular, measurements of excitation functions of the $p\left({ }^{6} \mathrm{He},{ }^{6} \mathrm{He}\right) p$ elastic scattering can provide information about states of the compound nucleus ${ }^{7} \mathrm{Li}$ in a region of excitation energies above $Q_{f u s}=9.975 \mathrm{MeV}$. We performed measurements of the elastic scattering excitation function in the range $E_{\text {exc }}^{7} \mathrm{Li}=10.4-11.7 \mathrm{MeV}$. In this region, the ${ }^{7} \mathrm{Li}$ has an interesting excited state at 11.24 $\mathrm{MeV}\left(J^{\pi}=3 / 2^{-}, T=3 / 2\right)$ which corresponds to the IAS (Isobaric Analog States) of the ${ }^{7} \mathrm{He}$ ground state.

\section{Experimental Setup}

The experiment has been performed at the 8MV Pelletron accelerator of the University of São Paulo using the RIBRAS $[2,3]$ system. The RIBRAS system is presently the only experimental equipment in South America capable of producing secondary beams of rare isotopes. The primary ${ }^{7} \mathrm{Li}$ beam of $300-500 \mathrm{nA}$ was accelerated to an energy of $24 \mathrm{MeV}$ and the ${ }^{9} \mathrm{Be}\left({ }^{7} \mathrm{Li},{ }^{6} \mathrm{He}\right){ }^{10} \mathrm{~B}$ reaction was used to produce the ${ }^{6} \mathrm{He}$. The secondary ${ }^{6} \mathrm{He}$ beam was produced in the RIBRAS ${ }^{9} \mathrm{Be}$ primary target $[2,3]$

\footnotetext{
a e-mail: ruben.pampa@gmail.com

be-mail: rubens@if.usp.br
} 
and its intensity was of about $10^{3} \mathrm{pps}$ on the $\mathrm{CH}_{2}$ secondary target after the second solenoid. A 12 $\mathrm{mg} / \mathrm{cm}^{2} \mathrm{CH}_{2}$ foil was used as degrador in the midway scattering chamber to improve the beam purity from $16 \%$ in the mid-scattering chamber to $92 \%$ in the secondary scattering chamber(see figure 1). The $12 \mathrm{mg} / \mathrm{cm}^{2} \mathrm{CH}_{2}$ secondary target was thick enough to fully stop the $11.5 \mathrm{MeV}^{6} \mathrm{He}$ beam with
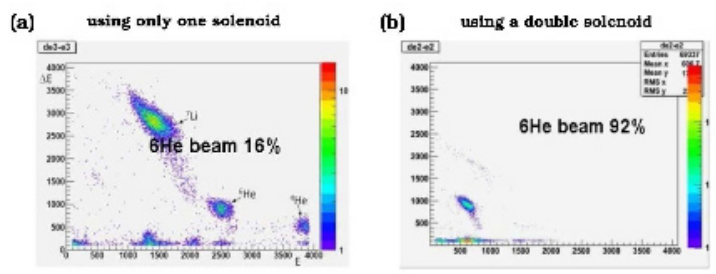

Figure 1. Spectra $\Delta E-E$ in the mid-scattering chamber (a) and in the secondary scattering chamber (b). A degrador was used in the mid-scattering chamber to improve the purity of the secondary ${ }^{6} \mathrm{He}$ beam from $16 \%$ to $92 \%$.

a energy resolution of $1 \mathrm{MeV}$ (FWHM). Two silicon E- $\Delta \mathrm{E}$ telescopes have been used to detect and identify the light particles coming out from reactions in the $\mathrm{CH}_{2}$ target. A $15 \mathrm{mg} / \mathrm{cm}^{2}$ pure carbon target (natural $\mathrm{C}$ target) was used to measure the background of reactions with the carbon and subtract it from the $\mathrm{CH}_{2}$ target spectrum.

\section{Thick Target Method}

With the present intensities of secondary beams, measurements of excitation functions with small energy steps would be very time consuming. The thick target method, consists of using thick sheets of polyethylene $\mathrm{CH}_{2}$ to stop the secondary beam (see figure 2). The scattering can take place at any position in the ${ }^{6} \mathrm{He}$ range and the energy of the recoil protons is related to the energy of the ${ }^{6} \mathrm{He}$ particle at the scattering position. By measuring the energy spectrum of the recoil protons one gets the entire excitation from zero up to the beam energy in one shot, as long as the elastic scattering is the only process producing protons.

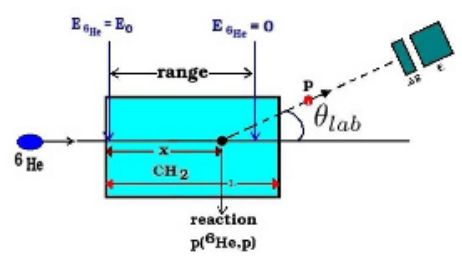

Figure 2. Illustration of thick target method. The reaction $\mathrm{p}\left({ }^{6} \mathrm{He}, \mathrm{p}\right)$ may happen in any point $x$ of the $\mathrm{CH}_{2}$ target and the recoil protons have to travel a distance $\frac{L-x}{\cos \theta_{l a b}}$, where $L$ is the total distance of the $\mathrm{CH}_{2}$ target.

\section{Results}

A $\Delta E-E$ spectrum for telescope 1 at 0 degrees is shown in figure 3(a). In this figure we can see the lines corresponding to $\alpha$ particles, triton, deuteron and proton nuclei from the $12 \mathrm{mg} / \mathrm{cm}^{2} \mathrm{CH}_{2}$ target. 
The insert (see figure 3(b)) shows a spectrum obtained with a pure $15 \mathrm{mg} / \mathrm{cm}^{2}$ carbon target. In figure 3 (c) we can see the proton line projection into energy-axis. The contribution of the carbon is shown by the dashed curve in this spectrum count $\times$ proton energy. No peaks are seen in the region around the resonance peak in the carbon spectrum.

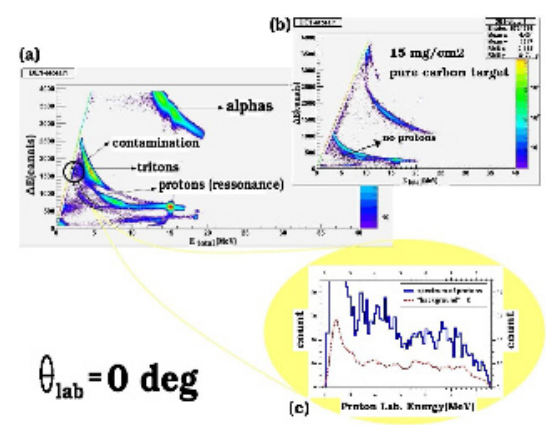

Figure 3. (a) $\Delta E-E$ spectrum for telescope 1 (b)Spectrum obtained with a pure $15 \mathrm{mg} / \mathrm{cm}^{2}$ carbon target. (c)Proton line projection into energy-axis.

The spectrum at $\theta_{l a b}=0$ degree presents a background in the region around the resonance peak. To overcome this problem we took the areas above and below the proton line near to the resonance region (see figure 4(a)) in such a way that we could estimate the background in the proton line, by taking an average between the counts above and below the proton line (see figures 4(b) and (c)). The average count has been subtracted from the proton spectrum which could see in figure $4(\mathrm{~d})$. The

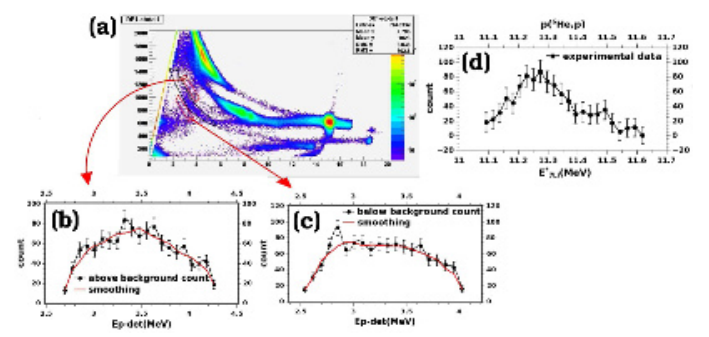

Figure 4. (a)Zoom of the biparametric spectrum for telescope 1. (b) and (c) are the projections of the background above and below the proton line. With this projections we could estimate approximately the background to be discounted from the proton spectrum. The results of this procedure is shown in (d) where we show the energy spectrum of the proton line with the background discounted.

biparametric spectrum for telescopes in 0, 20, and 25 degrees are shown in figure 5(a). In figure 5(b) we see the excitation functions corresponding to the proton line for the angles in which we worked. The excitation functions have been fitted by a Breit-Wigner function folded with a gaussian whose width is the experimental resolution $\sigma_{c . m}^{\text {exp.res. }}=60 \mathrm{keV}$. The fitting parameters are shown in table 1 . In addition, two R-matrix calculations have also been performed, one considering only $(p, p)$ channel (dotted line in figure 5(b)) and another including also the $(p, n)$ reaction (dashed line in figure 5(b)) which was measured in [4]. 

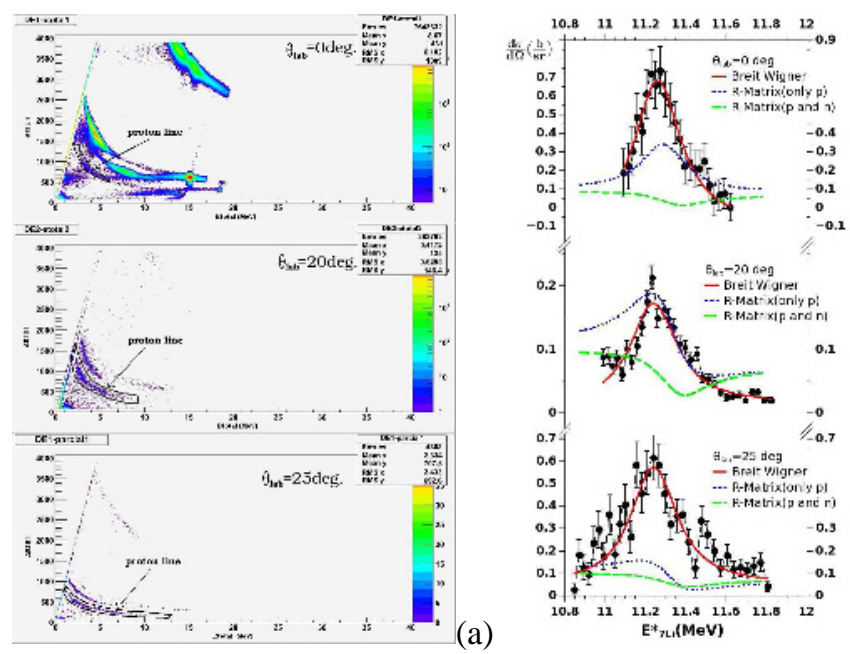

(b)

Figure 5. (a)Biparametric spectrum for telescopes in $\theta_{\text {lab }}^{\text {proton }}=0^{\circ}, 20^{\circ}, 25^{\circ}$ (b)Excitation functions. R-matrix calculations by P. Descouvemont $[5,6]$.

Table 1. Resonance parameters extracted by using Breit-Wigner function

\begin{tabular}{llll}
\hline$\theta_{\text {lab }}$ & $N=\gamma_{p}^{2}$ & $\Gamma(\mathrm{keV})$ & $E_{r}(\mathrm{MeV})$ \\
\hline $0^{\circ}$ & $0.052 \pm 0.004$ & $254 \pm 24$ & $11.11 \pm 0.02$ \\
$20^{\circ}$ & $0.011 \pm 0.001$ & $262 \pm 25$ & $11.28 \pm 0.02$ \\
$25^{\circ}$ & $0.036 \pm 0.001$ & $256 \pm 27$ & $11.11 \pm 0.03$ \\
\hline
\end{tabular}

\section{Conclusions}

We measured the $\mathrm{p}\left({ }^{6} \mathrm{He},{ }^{6} \mathrm{He}\right) \mathrm{p}$ elastic scattering excitation function at three angles $\theta_{\text {lab }}^{\text {proton }}=0,20$ and 25 degrees. We clearly see peaks in the position corresponding to the $11.24 \mathrm{MeV}$, state of the ${ }^{7} \mathrm{Li}$. A fit of the proton spectrum using a Breit-Wigner function shows that the peak has the expected energy and width at the three angles. R-matrix calculations give results that are in contradiction with the data. A reasonable agreement is obtained for the (p,p) one channel R-matrix calculation. However if one considers the (p,p) and (p,n) decay channels, the latter measured at [4], the calculations show an 'anti-peak' in the position of the (p,p) resonance, in contradiction with our data, whereas the (p,n) channel is well reproduced [4]. In this calculations we have used the partial widths reported in [4] for the (p,p) and (p,n) channels. The present results require futher investigation on the possible reasons to explain the disagreement between experimental data and R-matrix calculations.

\section{References}

[1] D.R. Tilley et al., Nucl. Phys. A708, 3(2002).

[2] R. Lichtenthäler et al., Eur. Phys. J. A 25, 733(2005).

[3] R. Lichtenthäler et al., AIP Conf. Proc. 1529,197-201(2013)

[4] G.V. Rogachev et al., Phys. Rev. Lett. 82, 3581 (2004).

[5] A.M. Lane and R.G. Thomas, Rev. Mod. Phys., 30, 257(1958).

[6] P. Descouvemont and D. Baye, Rep. Prog. Phys.73 036301(2010). 